Min Li, Tommaso Ranzani, Sina Sareh, Lakmal D. Seneviratne, Prokar Dasgupta, Helge A Wurdemann and Kaspar Althoefer 2014 Multi-Fingered Haptic Palpation utilizing Granular Jamming Stiffness Feedback Actuators Smart Mater. Struct. 23 095007 doi:10.1088/0964-1726/23/9/095007

\title{
Multi-Fingered Haptic Palpation utilizing Granular Jamming Stiffness Feedback Actuators
}

\author{
Min Li ${ }^{1}$, Tommaso Ranzani ${ }^{2}$, Sina Sareh ${ }^{1}$, Lakmal D. Seneviratne ${ }^{1,4}$, Prokar Dasgupta ${ }^{3}$, Helge A \\ Wurdemann ${ }^{1}$ and Kaspar Althoefer ${ }^{1}$ \\ ${ }^{1}$ Department of Informatics, Kings College London, London, WC2R 2LS, U.K. \\ ${ }^{2}$ BioRobotics Institute, Scuola Superiore Sant'Anna, Pisa, Italy. \\ ${ }^{3}$ Medical Research Council (MRC) Centre for Transplantation, King's College London, King's Health \\ Partners, Guy's Hospital, London SE1 9RT, UK. \\ ${ }^{4}$ College of Engineering, Khalifa University of Science, Technology and Research, Abu Dhabi, U.A.E. \\ E-mail: min.m.li@kcl.ac.uk; min.m.li0012@gmail.com
}

\begin{abstract}
This paper describes a multi-fingered haptic palpation method using stiffness feedback actuators for simulating tissue palpation procedures in traditional and robot-assisted minimally invasive surgery. Soft tissue stiffness is simulated by changing the stiffness property of the actuator during palpation. For the first time, granular jamming and pneumatic air actuation are combined together to realize stiffness modulation. The stiffness feedback actuator is validated by stiffness measurements in indentation tests and through stiffness discrimination based on a user study. According to the indentation test results, the introduction of a pneumatic chamber to granular jamming can amplify the stiffness variation range and reduce hysteresis of the actuator. The advantage of multi-fingered palpation using the proposed actuators is proven by the comparison of the results of the stiffness discrimination performance using two-fingered (sensitivity: $82.2 \%$, specificity: $88.9 \%$, positive predicative value: $80.0 \%$, accuracy: $85.4 \%$, time: $4.84 \mathrm{~s}$ ) and single-fingered (sensitivity: $76.4 \%$, specificity: $85.7 \%$, positive predicative value: $75.3 \%$, accuracy: $81.8 \%$, time: $7.48 \mathrm{~s}$ ) stiffness feedback.
\end{abstract}

\section{Introduction}

The hands of surgeons play an important role in tactile information acquisition for tumor identification during open surgery. In order to ensure that the entire tumor is removed and healthy tissue is spared as much as possible, the accurate localization of tumor is essential. Areas that are stiffer than the surrounding tissue are indicators for the presence of tumors. The texture and material properties of an object can be perceived by obtaining distributed pressure values during mechanical probing [1]. During Minimally Invasive Surgery (MIS), for the surgeon's hands to get directly in contact with tissue is not possible. Commonly, the soft tissue surface is prodded by a long surgical tool (a metal rod inserted through a trocar) to detect tumors; this process is called "instrument palpation" [2]. Nevertheless, instrument palpation is time consuming and not effective for small and deeply buried tumors since tactile information is missing [3]. Robot-assisted MIS (RMIS) has many benefits over traditional MIS, including enhanced 3D vision and improved dexterity, making the procedure easier for the surgeon. However, due to the lack of the sense of touch and possibilities to conduct "instrument palpation", intra-operative tumor identification is difficult to achieve.

Researchers have tried to implement "instrument palpation" methods using an RMIS system equipped with force feedback [4], however, this type of palpation procedure is not time-efficient [4]. Low-cost visual tactile cues can be introduced to compensate for the lack of tactile sensation - methods whereby material property distribution graphically overlaid over the image of the operating site are presented [3,5]. Nevertheless, graphically overlaying real-time stiffness data on top of the camera image can negatively impact on the clarity of the perceived image. 
Min Li, Tommaso Ranzani, Sina Sareh, Lakmal D. Seneviratne, Prokar Dasgupta, Helge A Wurdemann and Kaspar Althoefer 2014 Multi-Fingered Haptic Palpation utilizing Granular Jamming Stiffness Feedback Actuators Smart Mater. Struct. 23 095007 doi:10.1088/0964-1726/23/9/095007

Tactile actuators, which provide the user with a more intuitive experience of palpation than visual tactile cues, have been introduced for tumor identification in MIS as for instance described in [6]. Currently, tactile feedback systems employ various actuation technologies including tactile pin displays [7], vibrotactiles [8], pneumatically activated tactile displays [9], microfluid-based tactile displays [10], surface acoustic waves [11], focused ultrasound [12], electrorheologically-activated displays [13] and electrotactiles [14]. These devices can be classified into two main types based on the actuation principles: devices with movable components and devices based on materials with adjustable stiffness.

Providing distributed pressure (tactile information) to one finger during palpation has been conducted in $[7,9,15]$. Pneumatic tactile displays use air pressure to displace the skin, either by discharging air directly through nozzles against the skin or by inflating conformable tactors. Kim et al. [15] proposed and experimentally validated a pneumatic approach using an array of open nozzles $(5 \times 5)$ to discharge compressed air directly against the skin. Culjat et al. [9] developed a pneumatic balloon tactile display $(3 \times 2)$, which can be easily attached to existing commercial robot-assisted surgery systems such as da Vinci. Klein et al. [13] described a tactile actuator array (16 cells) using electrorheological fluid. Liu et al. [16] proposed a single MR fluid-based tactile element. Instead of only providing tactile feedback, Kim et al. [7] combines tactile pin display $(6 \times 8)$ with kinesthetic feedback in a palpation simulator. The experimental results show that compared to single-point force feedback, the single-fingered tactile palpation provides the user with more precise perception of the stiffness and shape of the embedded nodules. However, the development of aforementioned tactile devices is hampered by the complexity of control - all the elements of the actuator array need to be controlled simultaneously. The lack of commercially available tactile actuators limits current applications of tactile palpation simulation.

In order to reduce the control complexity imposed by tactile feedback devices, here, we propose the application of multi-fingered palpation feedback. Compared with tactile haptic methods as described in [7,9] for instance, the number of actuator elements in our multi-fingered palpation haptic system is reduced, leading to a decrease of fabrication costs and likelihood of mechanical failures. Palpation using more than one finger is common during traditional open surgery. Several works on multifingered palpation simulation have been reported in the literature. For instance, the Rutgers Master II force feedback glove was applied to training of knee palpation [17] and abdominal palpation for liver tumor detection [18]. These gloves use pneumatic actuators to apply forces to all fingertips of the user's hand, except for the small finger. However, the glove limits the range of motion to the fingers because of the pneumatic cylinders that are part of its structure. Another example is the Haptic Interface Robot (HIRO) device used for breast palpation simulation [19]. It consists of a force actuated 6-DOF robot arm and three fingers with 3-DOF force feedback. This device was upgraded to the five-fingered HIRO III device [20], however, its control system is still complex since each finger has more than one joint. Therefore, the price is relatively high and the device is bulky; it has a weight of about $3.8 \mathrm{~kg}$ and requires a control apparatus box which weights $23 \mathrm{~kg}$ and has a size of $443 \times 222 \times 464 \mathrm{~mm}^{3}$.

Although rendering force distribution in palpation using rigid movable components improves tumor identification results, these systems do not give the user an instinct stiffness feeling [6,7]. Hence, stiffness feedback has been introduced using materials with variable stiffness [13,16]. Stiffness control technologies can be divided into material stiffening and structural stiffening. The viscosity of 
Min Li, Tommaso Ranzani, Sina Sareh, Lakmal D. Seneviratne, Prokar Dasgupta, Helge A Wurdemann and Kaspar Althoefer 2014 Multi-Fingered Haptic Palpation utilizing Granular Jamming Stiffness Feedback Actuators Smart Mater. Struct. 23 095007 doi:10.1088/0964-1726/23/9/095007

electrorheological (ER) fluid can be adjusted by the application of an electric field. Similarly, the rheological properties of magnetorheological (MR) fluid can change when subjected to an external magnetic field. Prominent examples of the application of ER and MR in stiffness feedback are embodied in the work of Khaled et al. [13] and Liu et al. [16] who proposed a tactile actuator array using ER fluid and a single MR fluid-based tactile element, respectively. However, the controllability of these two methods is complex; it is difficult to tune stiffness and stable electric and magnetic fields are required to generate stiffness sensation. Moreover, the yielding strengths of ER fluid and MR fluid are only about $0-5 \mathrm{kPa}\left(5,000 \mathrm{~V} / \mathrm{mm}\right.$ at $\left.2-15 \mathrm{~mA} / \mathrm{cm}^{2}\right)$ and $0-100 \mathrm{kPa}(239 \mathrm{kA} / \mathrm{m}$ magnetic field) [21]. The physical phenomenon called granular jamming is a structural stiffening method for stiffness modulation. The jamming phenomenon is a type of phase transition of the granular matter in response to external stimuli [22]. Jamming can be induced by increasing density when a flexible membrane containing granular matter, e.g. coffee or rice, is vacuumed. The density can be controlled by regulating the vacuum level; thus it is possible to make particles act like a liquid, solid, or something in between. Its response time mainly depends on the vacuuming system. It has been used for haptic feedback [23].

This paper presents the design, simulation and experimental validation of a stiffness controllable multi-fingered haptic palpation method. Stiffness modulation is obtained through a granular jammingbased mechanism positioned on top of an inflatable structure for enhancing stiffness discrimination. A proof-of-concept study is performed to validate three main aspects:

1) granular jamming can be used for conveying stiffness information in remote palpation;

2) adding a pneumatic chamber below the granular jamming chamber enhances the performance of the stiffness modulation system;

3) multi-fingered palpation is superior to single-fingered palpation.

This article is organized as follows. Section 2 describes the design of the stiffness actuator and explains the concept of the multi-fingered haptic palpation system. An enhanced structure for the actuation system is presented and validated in Section 3. The ability of our actuators for stiffness modulation is validated using indentation tests in Section 4. Section 5 presents a user study of multifingered palpation using the proposed actuators. Section 6 concludes this paper.

\section{Stiffness feedback actuator and multi-fingered haptic palpation system}

In this study, granular jamming was exploited for stiffness control by regulating the vacuum level in the granular jamming chamber. Such strategy has already been validated in several robotic devices in the medical field [24-28]. Other researchers have shown that ground coffee is a suitable granule type for jamming [25]. Therefore, coffee was used inside our granular jamming chamber. The proposed pneumatic and granular jamming actuator is shown in Figure 1. The granular jamming chamber was made by filling $5 \mathrm{~g}$ of coffee powder (in our study, we used Lavazza, Qualità Rossa, medium roasting) in a latex membrane (average thickness: $0.07 \mathrm{~mm}$ ), which provides a relevant change in the elastic modulus during compression and has low hysteresis [29]. When the granular jamming chamber is activated (see Figure 1 (d)), the size of the chamber is reduced compared to the loose status as shown in Figure 1 (c). The particles will tend to adapt to the shape of the indenter (the finger in this case) as happens in the universal gripper where this phenomenon is exploited for gripping materials of different shapes [24]. In the envisaged use of the granular jamming based stiffening chamber, it is not desirable to have a permanent deformation of the granules when the finger is pressed against them. In 
Min Li, Tommaso Ranzani, Sina Sareh, Lakmal D. Seneviratne, Prokar Dasgupta, Helge A Wurdemann and Kaspar Althoefer 2014 Multi-Fingered Haptic Palpation utilizing Granular Jamming Stiffness Feedback Actuators Smart Mater. Struct. 23 095007 doi:10.1088/0964-1726/23/9/095007

order to avoid the permanent deformation, a pressurized pneumatic chamber was added below the granular jamming chamber. The pneumatic air chamber was made by pouring silicone $\left(\right.$ Ecoflex $^{\mathrm{TM}}$ 0050 - Smooth on Inc.) inside a printed mold produced by a 3D rapid prototype machine (ProJet ${ }^{\mathrm{TM}}$ HD 3000 Plus), which had a minimum layer resolution of $16 \mu \mathrm{m}$. During activation, air pressure is applied to inflate the top surface of the pneumatic chamber to guarantee the returning of the coffee to a flat shape and maintaining its contact with the fingertip during jamming (see Figure 1 (d)). Therefore this pneumatic chamber protects the granular material against shape change caused by the applied indentation force. When the applied contact force is removed, the pneumatic chamber bounces back, with the coffee powder in the granular jamming chamber being loose and freely movable.

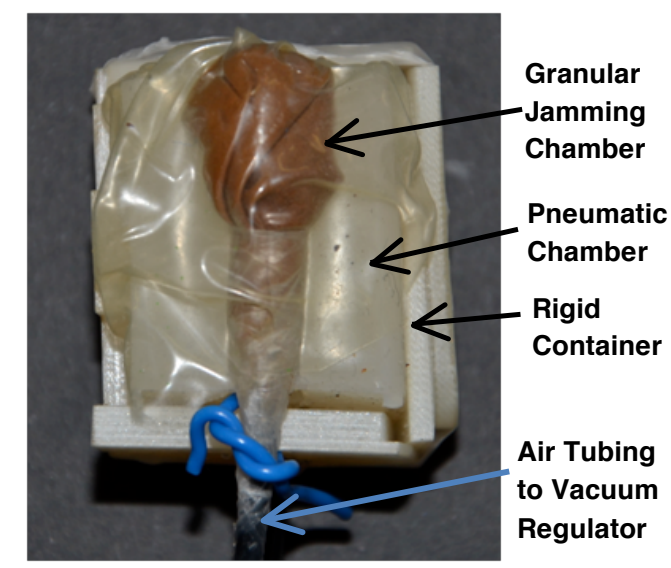

(a)

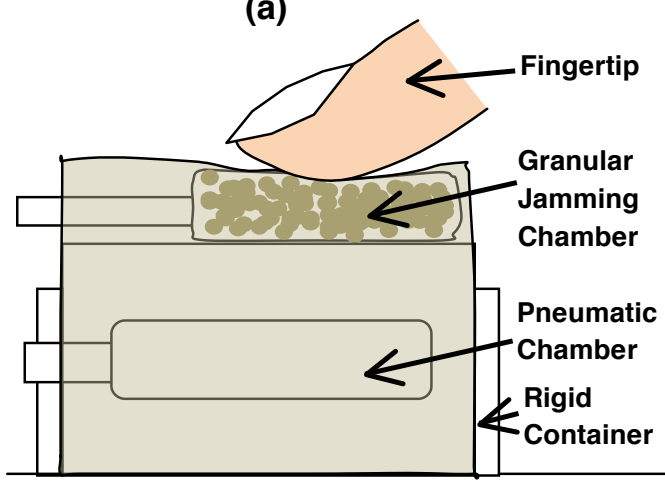

(c)

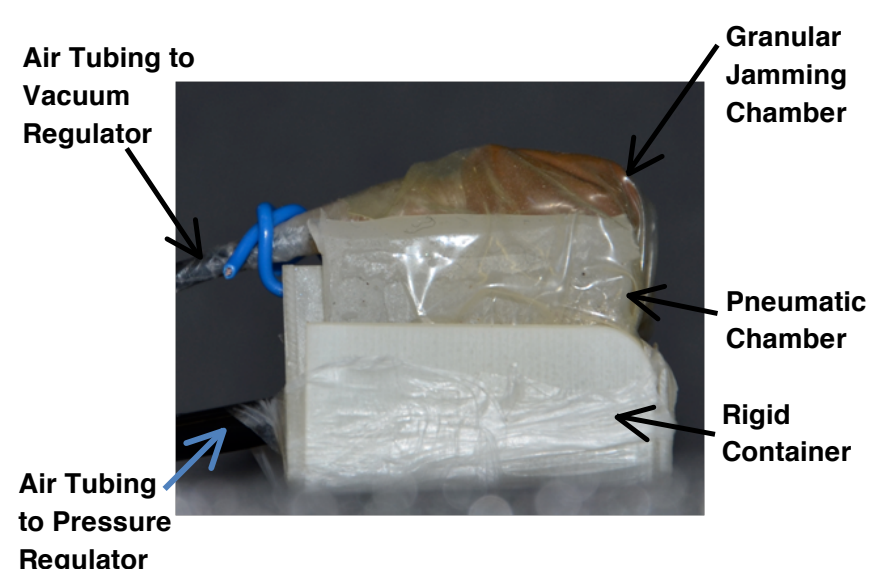

(b)

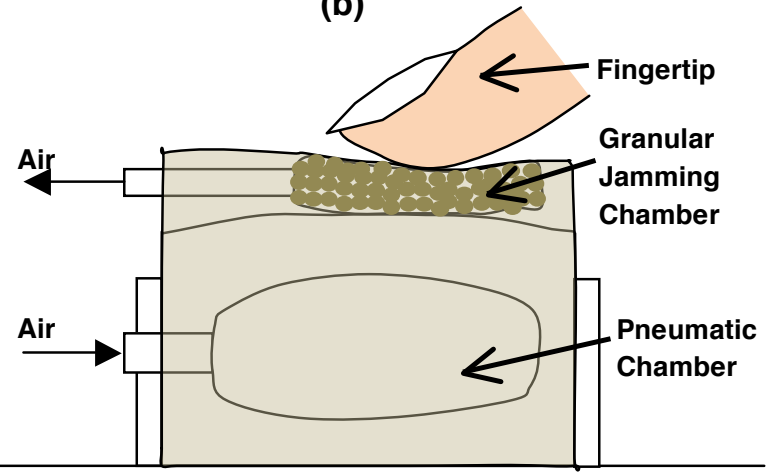

(d)

Figure 1. (a) Top and (b) side view of a prototype of pneumatic and granular jamming actuator and a profile view of the inactivated (c) and activated actuator (d).

A haptic device with two actuators was fabricated and integrated in the structure as depicted in Figure 2 to produce a two-fingered palpation system. Such structure provided a compact assembly of the two interfaces and limited the expansion of the silicone, during the air inflation, in all directions with the exception of the top surface, where the granular jamming chamber was placed. 
Min Li, Tommaso Ranzani, Sina Sareh, Lakmal D. Seneviratne, Prokar Dasgupta, Helge A Wurdemann and Kaspar Althoefer 2014 Multi-Fingered Haptic Palpation utilizing Granular Jamming Stiffness Feedback Actuators Smart Mater. Struct. 23 095007 doi:10.1088/0964-1726/23/9/095007

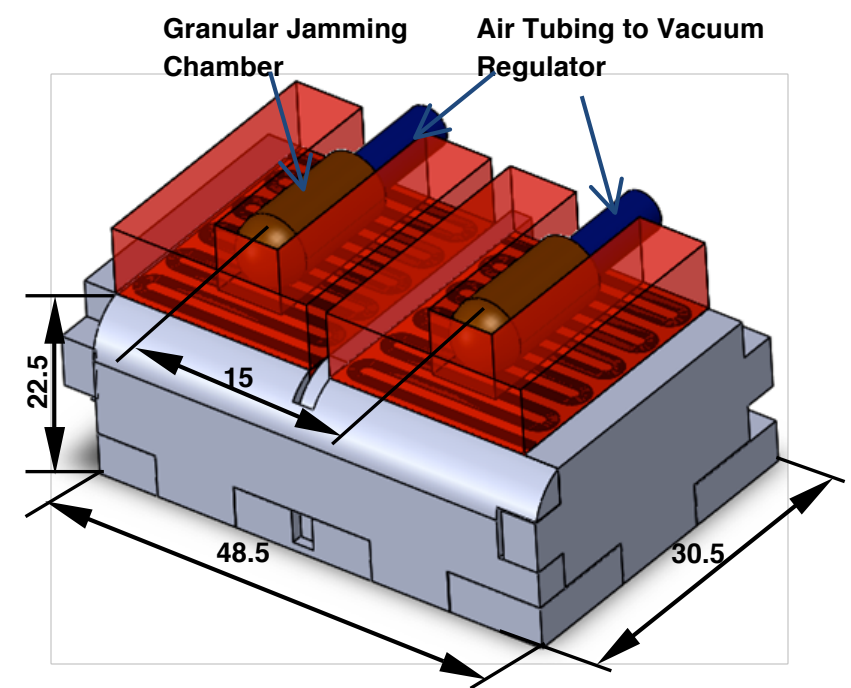

Figure 2. CAD model showing assembly of the two finger palpation system (units: $\mathrm{mm}$ )

Figure 3 (a) shows the block diagram of the control of the pneumatic haptic feedback actuators. According to the tactile sensing input (e.g. from the tele-manipulator), the air pressure values of the corresponding two channels can be calculated. In our evaluation study, predefined stiffness levels were used instead of the tactile sensing input. Pneumatic supply was provided by a compressor (BAMBI 150/500 air compressor) with an output of $0.15 \mathrm{MPa}$. Two NI DAQ cards (USB-6211) were used as analogue signal generators for the pressure regulators and vacuum regulators. The pressure regulators (SMC ITV0010) reduced the air pressure and inflated each of the actuators with proportional pressures ranging from 0 to $0.1 \mathrm{MPa}$. A Mastercool $90066-2 \mathrm{~V}-220$ pump and vacuum regulators (SMC ITV0090) were used to extract air from each of the actuators with proportional pressures ranging from -0.001 to $-0.1 \mathrm{MPa}$. A piece of non-woven fabric was used at the air tubing tip inside the granular jamming chamber to prevent coffee powder to enter into the tubing and a filter (ZFC050-04B, SMC) was used to further prevent particles to enter into the pump. Figure 3 (b) and (c) illustrate how the proposed actuators can be used in RMIS and MIS environments. Tissue stiffness information can be captured by the force and position sensors attached to the surgical tool at the slave side of the robot. At the master side, stiffness actuators are added to the control console to provide stiffness feedback to the fingers of the surgeon. 
Min Li, Tommaso Ranzani, Sina Sareh, Lakmal D. Seneviratne, Prokar Dasgupta, Helge A Wurdemann and Kaspar Althoefer 2014 Multi-Fingered Haptic Palpation utilizing Granular Jamming Stiffness Feedback Actuators Smart Mater. Struct. 23 095007 doi:10.1088/0964-1726/23/9/095007
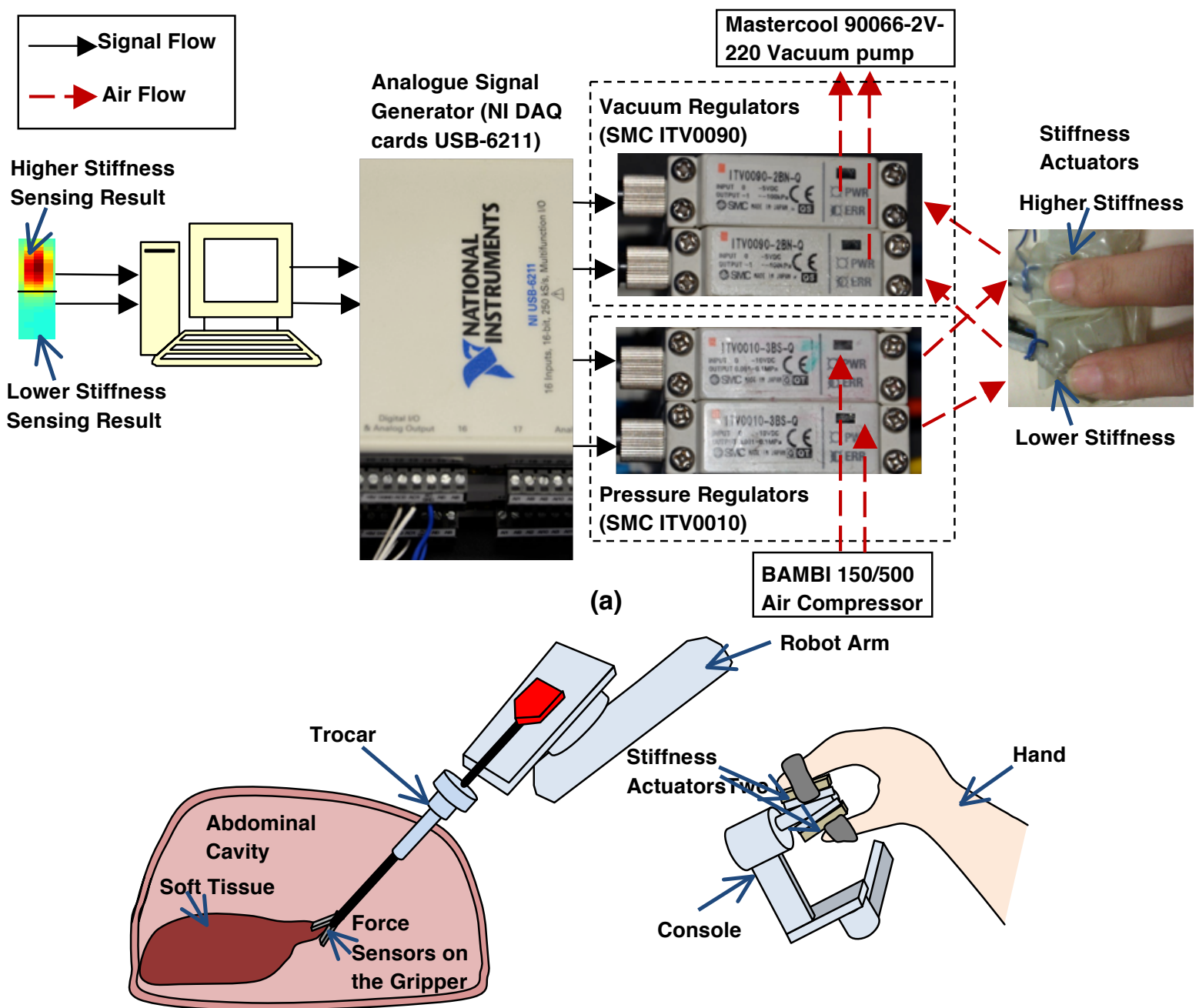

(b)

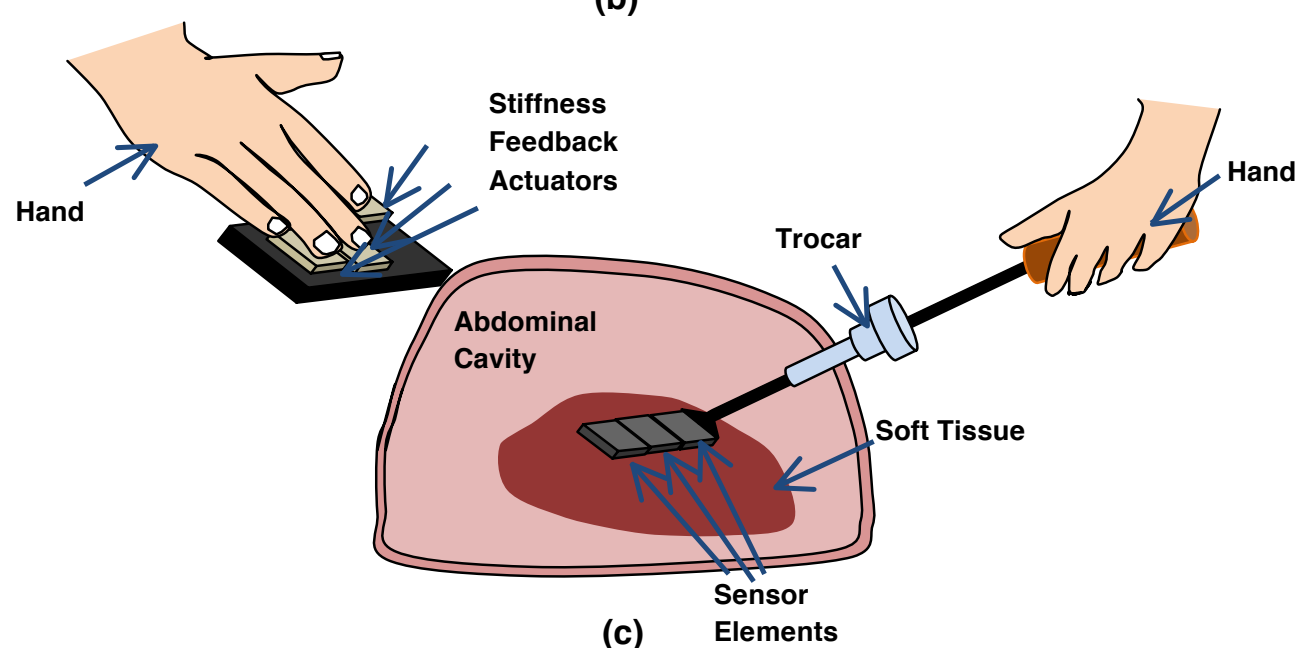

Figure 3. Schematic diagrams of (a) the multi-fingered palpation system and the applications in (b) an RMIS context and (c) a conventional MIS context.

\section{Structure enhancement}


Min Li, Tommaso Ranzani, Sina Sareh, Lakmal D. Seneviratne, Prokar Dasgupta, Helge A Wurdemann and Kaspar Althoefer 2014 Multi-Fingered Haptic Palpation utilizing Granular Jamming Stiffness Feedback Actuators Smart Mater. Struct. 23 095007 doi:10.1088/0964-1726/23/9/095007

According to our previous research about the pneumatic actuator inflation [30], the silicone membrane underwent a hemispherical deformation in response to the applied air pressure. In order to reduce the deformation and make the deformation distribution more even on the surface whilst still using a highly soft silicone, we propose a cotton thread to be embedded in a silicone layer above the inflatable chamber. As shown in Figure 4 (a), a groove in "OO" shape pattern was created on the top of the pneumatic chamber during the molding process of the silicone structure. A cotton thread was then placed inside the groove (see Figure 4 (b)). Another layer of silicone was constructed on top (see Figure 4 (c)). As shown in Figure 4 (d), a granular jamming chamber was then fixed on top. Finally a thin layer of latex membrane was used to cover the whole structure.

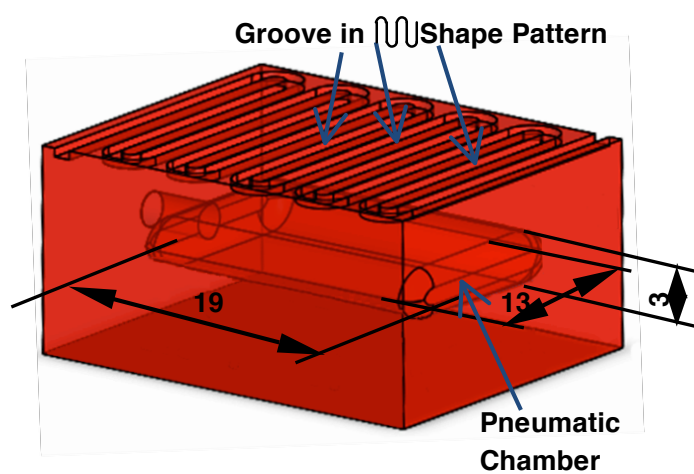

(a)

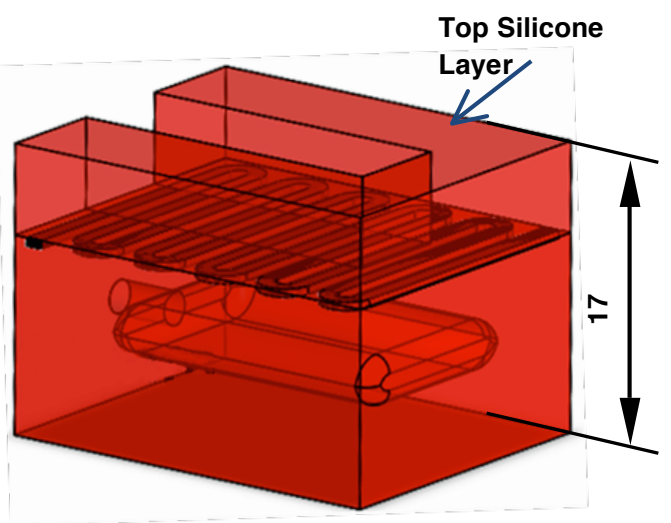

(c)

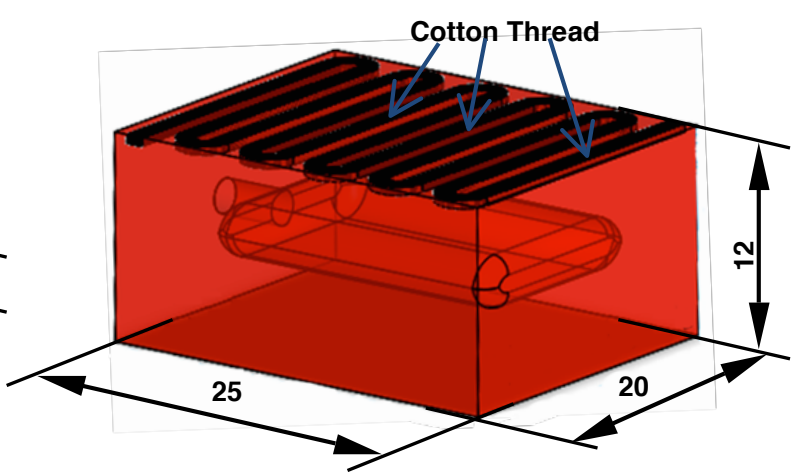

(b)

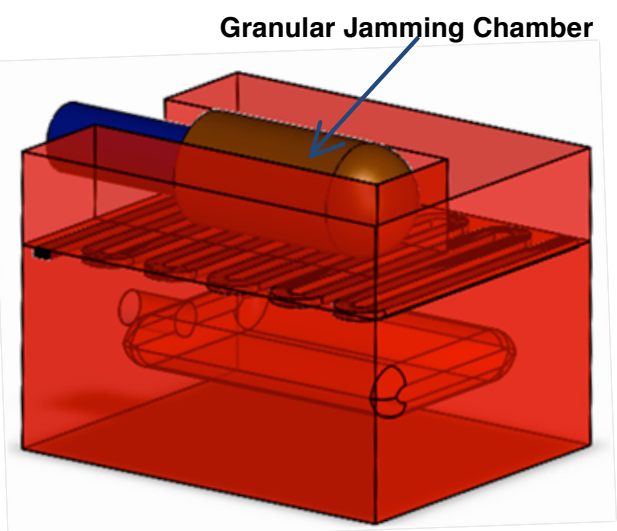

(d)

Figure 4. CAD model showing fabrication steps of the actuator (units: mm): (a) a groove on the top of the pneumatic chamber; (b) embedded cotton thread in the groove; (c) another silicone layer added; (d) a granular jamming chamber on top.

To validate the concept of using cotton thread for reducing the volumetric expansion of the pneumatic chamber and making the deformation distribution more even on the surface, the inflation behavior of the silicone pneumatic chamber was modeled using three-dimensional (3D) finite element (FE) modeling, as shown in Figure 5. A deformable fingertip model was placed above, contacting the top surface of the silicone pneumatic chamber. First, the simulation was run based on the cotton thread and the silicone structure. The material properties of the cotton thread and the silicone structure used in this FE model are shown in Table 1. Then, the material of the cotton thread was replaced by the silicone material to form a control condition. In other words, the simulation was run based on a silicone thread and the silicone structure in the control condition. In the simulation, a uniform 
Min Li, Tommaso Ranzani, Sina Sareh, Lakmal D. Seneviratne, Prokar Dasgupta, Helge A Wurdemann and Kaspar Althoefer 2014 Multi-Fingered Haptic Palpation utilizing Granular Jamming Stiffness Feedback Actuators Smart Mater. Struct. 23 095007 doi:10.1088/0964-1726/23/9/095007

distributed load (0.1 MPa) was applied on the inner surface of the air chamber. The lateral surfaces and the bottom of the chamber were fixed by an encastre boundary condition ("encastre" means fully constrained with no rotation or translation being allowed).

The fingertip was considered to have a width of $20 \mathrm{~mm}$ and a height of $18 \mathrm{~mm}$ representing a male index finger [31], shown in Figure 6. The cross section shape of the fingertip was obtained with reference to its anatomical images [32]. The cross section shape of the bone was set to be elliptical. The nail and bone were assumed to be linearly elastic. The Young's moduli of the nail and bone were set to be $170.0 \mathrm{MPa}$ and $17.0 \mathrm{GPa}$, respectively [33]. The Poisson's ratio was assumed to be 0.30 . The material parameters are listed in Table 2. The elastic behaviors of the finger's subcutaneous soft tissue and inner skin were simulated using a polynomial model [33]:

$$
U=\sum_{i+j=1}^{n} C_{i j}\left(\bar{I}_{1}-3\right)^{i}\left(\bar{I}_{2}-3\right)^{j}+\sum_{i=1}^{n} \frac{1}{D_{i}}(J-1)^{2 i},
$$

where $n, D_{i}, C_{i j}$ are the material parameters; $J$ is the elastic volume ratio; $\bar{I}_{1}$ and $\bar{I}_{2}$ are the first and second deviatoric strain invariants, respectively. The material parameters are listed in Table 3.

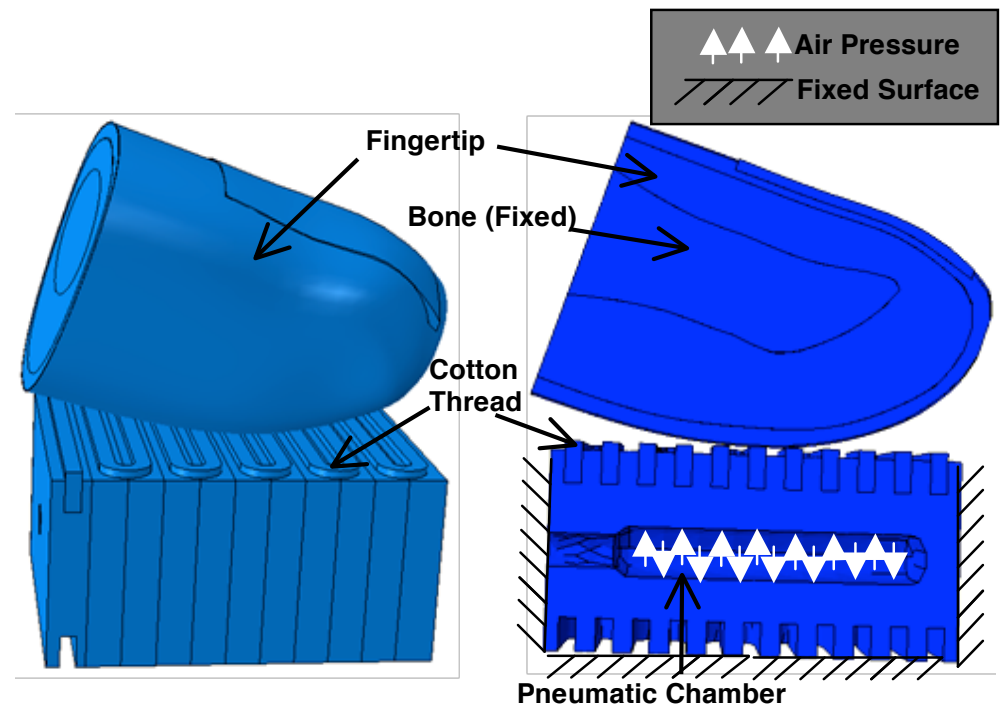

(a)

(b)

Figure 5. 3D modeling of the inflation behavior of the silicone pneumatic chamber with a fingertip contacting the top surface: (a) integral structure; (b) cross section.

Table 1. Material properties used in the finite element model

\begin{tabular}{lll}
\hline \multicolumn{1}{c}{ Properties } & Cotton thread & \multicolumn{1}{c}{ Silicone } \\
\hline Mass density (tonne $\left./ \mathrm{mm}^{3}\right)$ & $1.54 \times 10^{-9}[34]$ & $1.07 \times 10^{-9}[35]$ \\
Young's Modulus $(\mathrm{MPa})$ & $8200[34]$ & Null \\
Hyperelasticity & Null & Uniaxial test data \\
Poisson's ratio & 0.5 & 0.4 \\
\hline
\end{tabular}


Min Li, Tommaso Ranzani, Sina Sareh, Lakmal D. Seneviratne, Prokar Dasgupta, Helge A Wurdemann and Kaspar Althoefer 2014 Multi-Fingered Haptic Palpation utilizing Granular Jamming Stiffness Feedback Actuators Smart Mater. Struct. 23 095007 doi:10.1088/0964-1726/23/9/095007

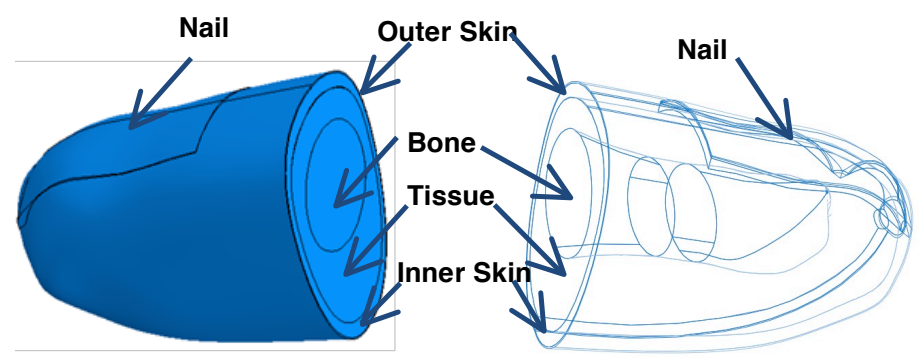

(a)

(b)

Figure 6. Fingertip model: shaded (a) and wireframe (b) render model.

Table 2. Elastic parameters for the soft tissues of the fingertip [31]

\begin{tabular}{llllll}
\hline & Bone & Nail & $\begin{array}{l}\text { Inner } \\
\text { skin }\end{array}$ & Outer skin & Soft tissue \\
\hline Density $\left(\mathrm{kg} / \mathrm{m}^{3}\right)$ & 2.7 & 2.0 & 1.0 & 1.0 & 1.0 \\
\hline
\end{tabular}

Table 3. Elastic parameters for the soft tissues of the fingertip [36]

\begin{tabular}{lllllll}
\hline & $\left.\mathbf{C}_{\mathbf{1 0}} \mathbf{( M P a}\right)$ & $\left.\mathbf{C}_{\mathbf{0 1}} \mathbf{( M P a}\right)$ & $\left.\mathbf{C}_{\mathbf{1 1}} \mathbf{( M P a}\right)$ & $\left.\mathbf{C}_{\mathbf{2 0}} \mathbf{( M P a}\right)$ & $\left.\mathbf{C}_{\mathbf{0 2}} \mathbf{( M P a}\right)$ & $\mathbf{D}_{\mathbf{1}}\left(\mathbf{M P a}^{\mathbf{- 1}}\right)$ \\
\hline Inner skin & $2.34 \mathrm{E}-3$ & $5.42 \mathrm{E}-3$ & -0.262 & 0.239 & $7.47 \mathrm{E}-2$ & 13.3 \\
Tissue & $5.97 \mathrm{E}-4$ & $1.34 \mathrm{E}-3$ & $-6.55 \mathrm{E}-2$ & $5.96 \mathrm{E}-2$ & $1.87 \mathrm{E}-2$ & 53.3 \\
\hline
\end{tabular}

Using the proposed FE models of the cotton thread and the silicone chamber, the inflation behavior of the silicone chamber was modeled. Figure 7 shows the simulation result when a deformable fingertip is in contact with the actuator surface. A more even deformation distribution on the surface of the actuator was achieved by incorporating networks of cotton thread. The enhanced structure reduced the deformation magnitude of the actuator surface almost by 5 times. Therefore, this network of cotton thread was integrated into our actuators and used in this study. 
Min Li, Tommaso Ranzani, Sina Sareh, Lakmal D. Seneviratne, Prokar Dasgupta, Helge A Wurdemann and Kaspar Althoefer 2014 Multi-Fingered Haptic Palpation utilizing Granular Jamming Stiffness Feedback Actuators Smart Mater. Struct. 23 095007 doi:10.1088/0964-1726/23/9/095007

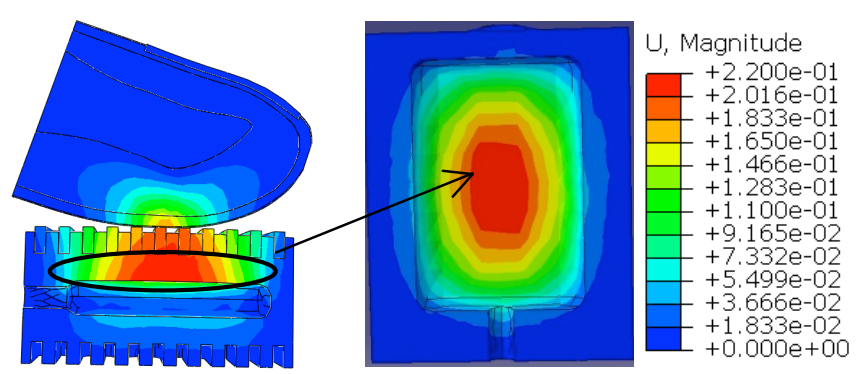

(a)

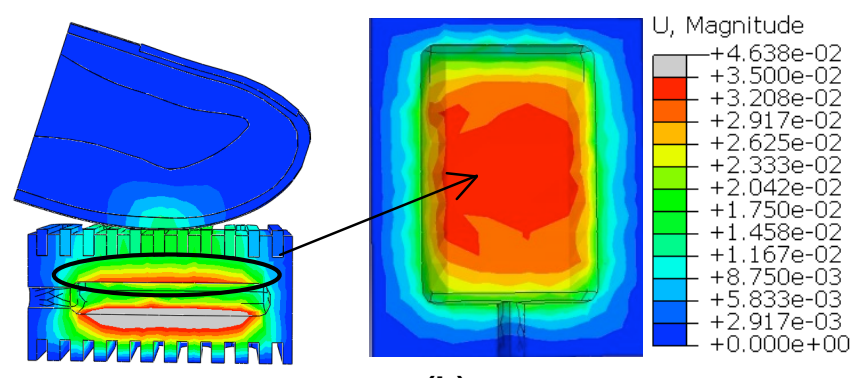

(b)

Figure 7. Deformation results: the deformable finger and actuator with no structure enhancement (a) and with structure enhancement (b).

\section{Stiffness modulation validation}

In order to validate the performance of the stiffness modulation using the proposed pneumatic and granular jamming actuator, an experiment was set up as shown in Figure 8. A rigid indenter was used to conduct the indentation test and the normal reaction force and indentation depth information was recorded. A pneumatic and granular jamming actuator was fixed at one side of a guide rail. An ATI Nano 17 F/T sensor (SI-12-0.12, resolution $0.003 \mathrm{~N}$ with 16-bit data acquisition card), which was attached to a hemispherical indenter for force measurement, was fixed to the sliding block on the guide rail. A Maxon EC-30 motor-powered linear module was used to control the indentation depth. A LabVIEW program was developed to control the motor position and record the indentation depth, air pressure, vacuum level, and force data.

Tests were conducted at $3 \mathrm{~mm}$ indentation depth and using different combinations of pressure inside the chamber and vacuum levels in the granular jamming-based stiffening chamber. A maximum pressure of $0.02 \mathrm{MPa}$ was chosen in order to maintain a small amount of deformation of the air chamber surface. The maximum vacuum pressure was $-0.1 \mathrm{MPa}$. The indentation was performed at a very low speed of $0.1 \mathrm{~mm} / \mathrm{s}$ in order to neglect possible dynamical effects. Each test was repeated 8 times. 
Min Li, Tommaso Ranzani, Sina Sareh, Lakmal D. Seneviratne, Prokar Dasgupta, Helge A Wurdemann and Kaspar Althoefer 2014 Multi-Fingered Haptic Palpation utilizing Granular Jamming Stiffness Feedback Actuators Smart Mater. Struct. 23 095007 doi:10.1088/0964-1726/23/9/095007

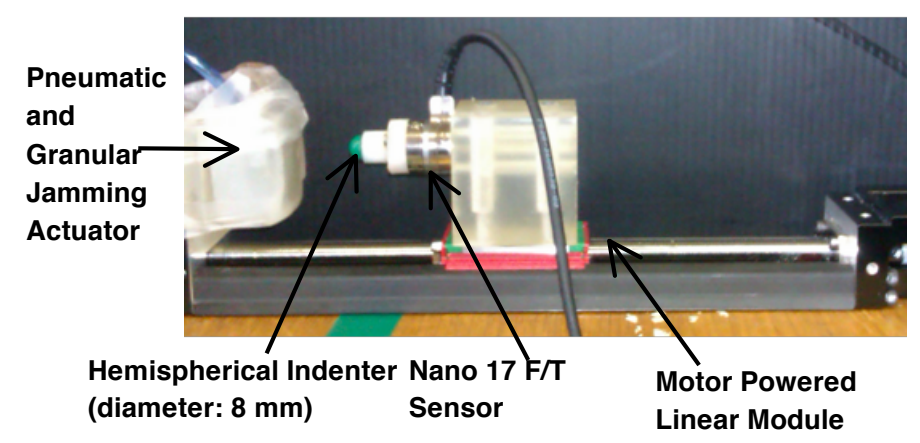

Figure 8. Experiment setup of stiffness modulation validation.

Figure 9 (a) presents the reaction force from the actuator during the indentation tests when only the pneumatic chamber was activated and the granular jamming chamber was present but not vacuumed. It is evident that the increase in the pressure level increased the stiffness of the actuator, but it would be very difficult to discriminate the stiffness levels since the curves corresponding to pressure values greater than $0 \mathrm{MPa}$ are virtually overlapping. In addition, as evident from Figure 9 (a), the hyperelastic behavior of the silicone material was dominant. Figure 9 (b) shows the reaction force from the actuator during the indentation tests when three levels of air pressure $(0.0 \mathrm{MPa}, 0.015 \mathrm{MPa}$, and $0.02 \mathrm{MPa})$ and three levels of vacuum pressure $(0.0 \mathrm{MPa},-0.03 \mathrm{MPa}$, and $-0.1 \mathrm{MPa})$ were applied. As expected, we can observe that higher vacuum pressures (i.e. when the granular jamming chamber was activated) produced steeper slopes of the stress-strain curves. The actuator had an almost linear response with the exception of the very first tract, when none of the two chambers were activated, and thus, the hyperelasticity of the silicone material was dominant. The inflation of air in the pneumatic chamber affected mainly the slope of the curves and tended to increase the distance between the curves corresponding to different vacuum levels. The maximum reached force, however, was not increased considerably by the air pressure inside the pneumatic chamber since this pneumatic chamber absorbed part of the load and thus avoided permanent deformation of the variable stiffness chamber. Referring to the test results shown in Figure 9 (a) and (b), one can see that the stiffness modulation range was amplified by the inflation of air, creating distinctive force profiles.

Figure 9 (c) depicts the stiffness variation profile calculated applying Hook's law to the curves of Figure 9 (b). One of the main advantages of the proposed actuator, as evident from Figure 9 (c) is that the pressurized actuator presented a more linear change in stiffness at the different vacuum levels. In contrast, the change in stiffness was more abrupt when no air pressure was applied. When the air pressure was $0.02 \mathrm{MPa}$, the relationship between stiffness and vacuum pressure was more linear and the hysteresis was lower than when the other two air pressure levels were applied. The hysteresis was computed as the enclosed area between the unloading and the loading cycles. As shown in Figure 9 (d) hysteresis was decreased significantly (up to $65 \%$ less) when the air chamber was inflated confirming that the permanent deformation of the granular jamming chamber was considerably reduced. Therefore, $0.02 \mathrm{MPa}$ air pressure was applied in the following user study. 
Min Li, Tommaso Ranzani, Sina Sareh, Lakmal D. Seneviratne, Prokar Dasgupta, Helge A Wurdemann and Kaspar Althoefer 2014 Multi-Fingered Haptic Palpation utilizing Granular Jamming Stiffness Feedback Actuators Smart Mater. Struct. 23 095007 doi:10.1088/0964-1726/23/9/095007

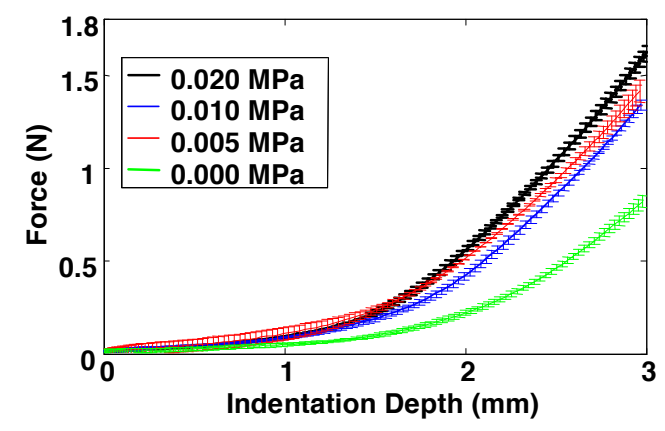

(a)

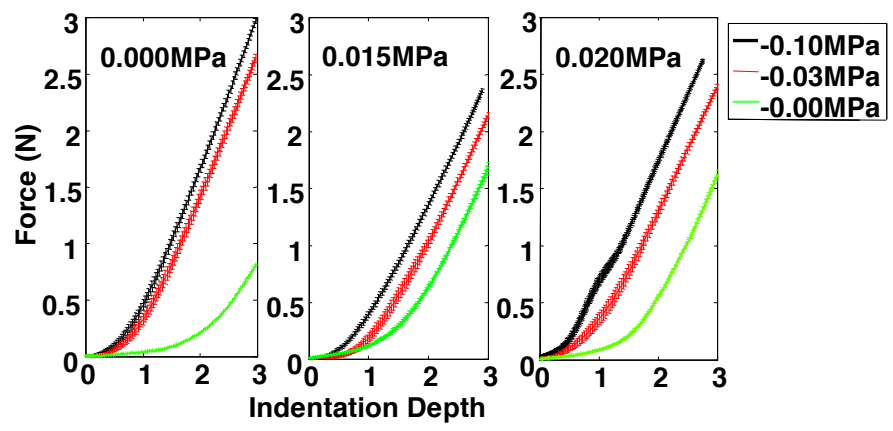

(b)

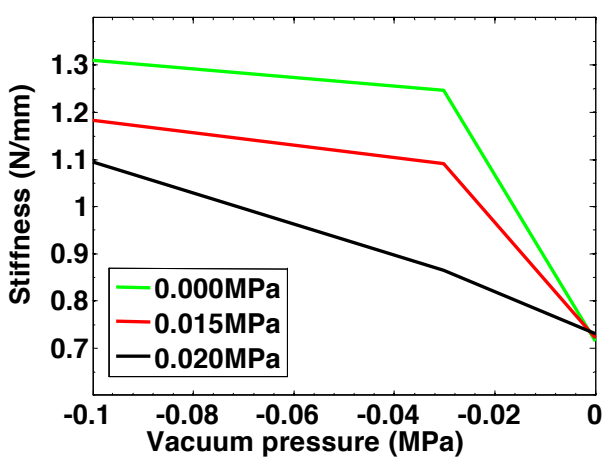

(c)

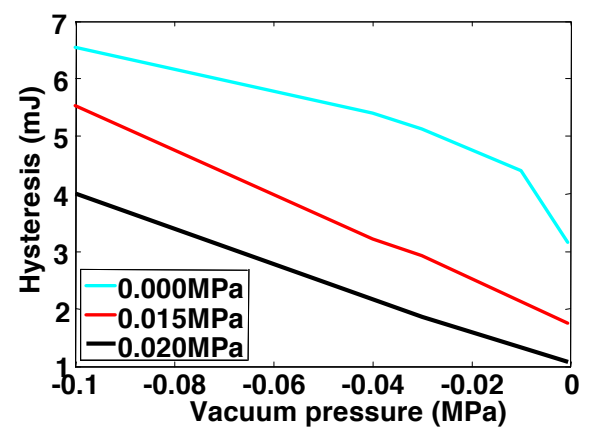

(d)

Figure 9. Indentation result with error bar when only the pneumatic chamber in the actuator is activated (a); indentation result with error bar when both the pneumatic chamber and granular jamming chamber in the actuator are activated (b); stiffness variation profile when both the pneumatic chamber and granular jamming chamber in the actuator are activated (c); hysteresis when both the pneumatic chamber and granular jamming chamber in the actuator are activated (d).

\section{User study}

A user study of stiffness discrimination was conducted to validate the ability of tissue stiffness interpretation of the proposed pneumatic and granular jamming actuators. Two types of feedback were investigated, single-fingered feedback and two-fingered feedback. Three levels of vacuum were used $-0,-0.01,-0.1 \mathrm{MPa}$. Experiencing higher stiffness of the actuator underneath a finger was considered as an indication of a tumor; receiving the same stiffness level by two fingers was considered as an indication for no tumor present. Eight types of combinations of stiffness levels were studied. During the single-fingered palpation, the participants were presented with two levels of stiffness in order, while their two fingers were fed back with the two levels of stiffness simultaneously during the two-fingered palpation. During the test a stopwatch (with a measurement precision of $\pm 1 \mathrm{~s}$ ) was used in order to measure the time required by the participant to explore the surface of each trial. Twelve participants were involved in the trials: 4 women and 8 men. The demographics of the involved participants are presented in Table 4 . All the tests were performed pseudo-randomly by each participant.

Table 4. Overview of demographics and experience of the participants 
Min Li, Tommaso Ranzani, Sina Sareh, Lakmal D. Seneviratne, Prokar Dasgupta, Helge A Wurdemann and Kaspar Althoefer 2014 Multi-Fingered Haptic Palpation utilizing Granular Jamming Stiffness Feedback Actuators Smart Mater. Struct. 23 095007 doi:10.1088/0964-1726/23/9/095007

\begin{tabular}{ll}
\hline Item & Detail \\
\hline Age range & $23-36$ \\
Average age & 28.7 \\
Gender & $\circ: 4 ; \Im: 8$ \\
Handedness & R: $12 ; \mathrm{L}: 0$ \\
Palpation experience & 0 \\
Engineering background & 12 \\
VR simulator & 0 \\
\hline
\end{tabular}

The following statistical measures were used to evaluate the performances of the investigated feedback modes. Sensitivity $\mathrm{Se}$ [37], which is a measure of the test's ability to identify positive results, was defined as:

$$
S e=\sum_{i=1}^{n} T P_{i} / \sum_{i=1}^{n}\left(T P_{i}+F N_{i}\right)
$$

where $n$ is the number of trials; TP is true positives - participants claim there is a hard nodule when there is one; $F N$ is false negatives - participants claim there is no hard nodule when there is actually one.

Specificity $S p$ [37], a measure of the test's ability to correctly identify negative results, was defined as:

$$
S p=\sum_{i=1}^{n} T N_{i} / \sum_{i=1}^{n}\left(T N_{i}+F P_{i}\right)
$$

where $T N$ is true negatives - participants claim there is no hard nodule when there is no one; $F P$ is false positives - participants claim there is a hard nodule when there is actually no one.

Positive predictive value $P P V$ [38], or precision rate, was defined by the following formula:

$$
P P V=\sum_{i=1}^{n} T P_{i} / \sum_{i=1}^{n}\left(T P_{i}+F P_{i}\right)
$$

Accuracy $A C C[38]$ is defined as:

$$
A C C=\sum_{i=1}^{n}\left(T P_{i}+T N_{i}\right) / \sum_{i=1}^{n}\left(T N_{i}+T N_{i}+F P_{i}+F N_{i}\right) .
$$

Wilson score intervals [39], which were calculated for those statistical measures at $95 \%$ confidence level, are defined using the following formula:

$$
\frac{1}{1+\frac{z^{2}}{n}}\left[\hat{p}+\frac{z^{2}}{2 n} \pm z \sqrt{\frac{\hat{p}(1-\hat{p})}{n}+\frac{z^{2}}{4 n^{2}}}\right],
$$

where $n$ is the sample size; $\hat{p}$ is the proportion of successes estimated from the statistical sample; $z$ is the $1-\alpha / 2$ percentile of a standard normal distribution where $\alpha$ is the error percentile. Here, since the confidence level is $95 \%$, the error $\alpha$ is $5 \%$.

The differences between the two-fingered palpation and single-fingered palpation on $S e, S p, P P V$ and $A C C$ were examined by comparing the observed probabilities $\left(p_{1}\right.$ and $\left.p_{2}\right)$ with a combined interval $(C I)$, defined as [40]:

$$
C I=\sqrt{\left(P_{1}-p_{1}\right)^{2}+\left(P_{2}-p_{2}\right)^{2}},
$$


Min Li, Tommaso Ranzani, Sina Sareh, Lakmal D. Seneviratne, Prokar Dasgupta, Helge A Wurdemann and Kaspar Althoefer 2014 Multi-Fingered Haptic Palpation utilizing Granular Jamming Stiffness Feedback Actuators Smart Mater. Struct. 23 095007 doi:10.1088/0964-1726/23/9/095007

where if $p_{1}<p_{2}, P_{1}$ is the upper bound of $p_{1}$ and $P_{2}$ is the lower bound of $p_{2}$. When $\left|p_{1}-p_{2}\right|>C I$, there is a significant difference between the two tests.

Figure 10 presents the sensitivities $S e$, specificities $S p$, positive predictive values $P P V$, and accuracies $A C C$ with Wilson score intervals at a $95 \%$ confidence level of the stiffness level discrimination tests. The population was 192 ( 2 values $\times 8$ trails $\times 12$ participants). From Figure 10 one can see that twofingered feedback has higher values of $S e, S p, P P V$ and $A C C$. However, the differences are not significant (see Table 5).

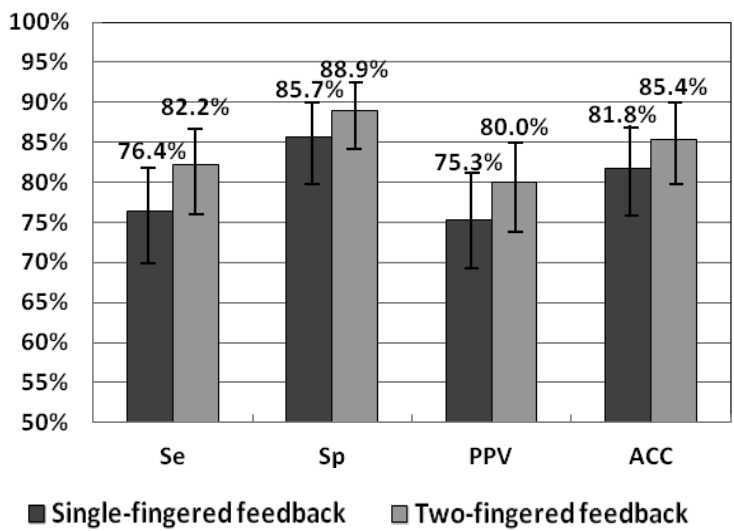

Figure 10. The sensitivities, specificities, positive predictive values, and accuracies of stiffness level discrimination with Wilson score intervals at a 95\% confidence level.

Table 5. Comparison of sensitivity, specificity, and accuracy in stiffness level discrimination tests of single-fingered feedback and two-fingered feedback

\begin{tabular}{lccc}
\hline Item & Combined interval $(\boldsymbol{C I})$ & Probability difference $(\boldsymbol{\Delta p})$ & Significance \\
\hline $\boldsymbol{S e}$ & 0.081 & 0.058 & $C I>\Delta p$, No \\
$\boldsymbol{S p}$ & 0.067 & 0.032 & $C I>\Delta p$, No \\
$\boldsymbol{P P} \boldsymbol{V}$ & 0.083 & 0.047 & $C I>\Delta p$, No \\
$\boldsymbol{A C C}$ & 0.074 & 0.036 & $C I>\Delta p$, No \\
\hline
\end{tabular}

Figure 11 presents the consumed time during the tests of stiffness level discrimination. Since the sample size was as large as 96 ( 8 trails $\times 12$ participants), it can be considered as normally distributed and a student $t$-test was performed to compare the consumed time during the tests. The two-fingered feedback test consumed significantly less time than the single-fingered feedback test since $p$-value was $2.60 \times 10^{-14}$.

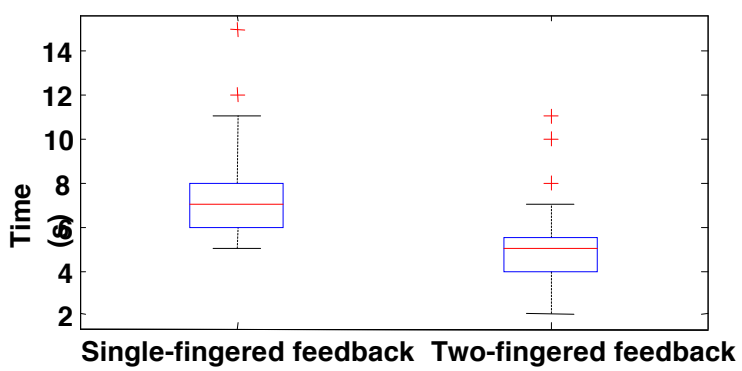


Min Li, Tommaso Ranzani, Sina Sareh, Lakmal D. Seneviratne, Prokar Dasgupta, Helge A Wurdemann and Kaspar Althoefer 2014 Multi-Fingered Haptic Palpation utilizing Granular Jamming Stiffness Feedback Actuators Smart Mater. Struct. 23 095007 doi:10.1088/0964-1726/23/9/095007

Figure 11. The consumed time during the tests of stiffness level discrimination of single-fingered feedback and two-fingered feedback.

\section{Discussion}

In a multi-fingered haptic system, the actuators when actuated by equal amounts of input pressure must output equal levels of stiffness, requiring precise fabrication of identical granular jamming chambers with equal shape and capacity. In this paper, they were hand-made, and it was found difficult to produce completely identical granular chambers. This difficulty can be overcome by a standardized fabrication process.

As we observed during the stiffness modulation tests, the activated actuators had an almost linear response thanks to the activated granular jamming chamber and the pneumatic chamber while the inactivated actuator showed the characteristics of the hyperelastic silicone material of the actuator. The inflation of air in the pneumatic chamber amplified the stiffness variation range by affecting the slope of the curves and tended to increase the distance between the curves corresponding to the different vacuum levels. It should be mentioned that part of the indentation load was absorbed by the pneumatic chamber, which helps avoiding permanent deformation of the variable stiffness chamber.

Some hysteresis can be observed between the stress-strain curves of loading and unloading especially when the vacuum level was higher than $-0.06 \mathrm{MPa}$ (see Figure 9 (d)). By introducing the air chamber under the granular jamming chamber, hysteresis was decreased considerably (by up to 65\%) confirming that the permanent deformation of the granular jamming chamber was considerably reduced. To further improve the performance of the system, hysteresis compensation algorithms should also be considered for future studies.

The two-fingered feedback showed higher values of $S e, S p, P P V$ and $A C C$ than the single-fingered feedback. However, the differences were not significant. Increasing the number of actuators to three may enlarge the significance; this needs more research. The two-fingered feedback test consumed significantly less time than the single-fingered feedback test. Therefore, the user study results of discrimination of stiffness levels reveal that the two-fingered feedback is more time-efficient to convey tissue stiffness information to the user. By an extension of this logic, multi-fingered feedback is more time-efficient to convey tissue stiffness information to the user than single-fingered feedback.

\section{Conclusion}

In this paper, a multi-fingered palpation method using a pneumatic and granular jamming actuator, which simulates tissue stiffness directly, is proposed. This principle is proven by examining the stiffness variability of the actuators and evaluating their performance in discriminating stiffness levels exploring the perception of single-fingered and two-fingered feedback in a user study. The experimental results show that the stiffness of the actuator can be controlled to simulate tissue stiffness; the introduction of a pneumatic chamber to granular jamming can amplify the stiffness variation range and reduce hysteresis of the actuator; the two-fingered feedback using the proposed pneumatic and granular jamming actuators is more time-efficient with regards to the discrimination of stiffness levels than what can be achieved using single-fingered feedback. The proposed pneumatic and granular jamming actuators provide a solution for multi-fingered palpation haptics. The accuracy and time-efficiency advantages of using multi-fingered palpation over single-fingered palpation have 
Min Li, Tommaso Ranzani, Sina Sareh, Lakmal D. Seneviratne, Prokar Dasgupta, Helge A Wurdemann and Kaspar Althoefer 2014 Multi-Fingered Haptic Palpation utilizing Granular Jamming Stiffness Feedback Actuators Smart Mater. Struct. 23 095007 doi:10.1088/0964-1726/23/9/095007

been shown in this study. By incorporating real-time tactile sensing data, the application of these actuators can be extended from simulated haptics to intra-operative palpation haptics.

\section{Acknowledgement}

We thank both the engineering and medical community for their helpful discussions and comments. The work described in this paper is partially funded by the China Scholarship Council, the Seventh Framework Programme of the European Commission under grant agreement 287728 in the framework of EU project STIFF-FLOP, the Vattikuti Foundation, as well as by the National Institute for Health Research (NIHR) Biomedical Research Centre based at Guy's and St Thomas' NHS Foundation Trust and King's College London. The views expressed are those of the authors and not necessarily those of the NHS, the NIHR or the Department of Health.

\section{References}

[1] Schiff W, Foulke E. Tactual perception: a source book. 1982.

[2] Ottermo MV, Stavdahl O, Johansen T a. Palpation instrument for augmented minimally invasive surgery. IEEE/RSJ Int. Conf. Intell. Robot. Syst. (IEEE Cat. No.04CH37566), vol. 4, Ieee; 2004, p. 3960-4.

[3] Miller AP, Peine WJ, Son JS, Hammoud MDZT. Tactile imaging system for localizing Lung nodules during video assisted thoracoscopic surgery. Proc 2007 IEEE Int Conf Robot Autom 2007:2996-3001.

[4] Mahvash M, Gwilliam J, Agarwal R, Vagvolgyi B, Su L-M, Yuh DD, et al. Force-feedback surgical teleoperator: controller design and palpation experiments. 2008 Symp Haptic Interfaces Virtual Environ Teleoperator Syst 2008:465-71.

[5] Yamamoto T, Abolhassani N. Augmented reality and haptic interfaces for robot assisted surgery. Int J Med Robot Comput Assist Surg 2012;8:45-56.

[6] King C, Culjat MO, Franco ML, Bisley JW, Carman GP, Dutson EP, et al. A multielement tactile feedback system for robot-assisted minimally invasive surgery. Haptics, IEEE Trans 2009;2:52-6.

[7] Kim S-Y, Kyung K-U, Park J, Kwon D-S. Real-time area-based haptic rendering and the augmented tactile display device for a palpation simulator. Adv Robot 2007;21:961-81.

[8] McMahan W, Gewirtz J. Tool contact acceleration feedback for telerobotic surgery. IEEE Trans Haptics 2011;4:1939-1412.

[9] Culjat M, King C-H, Franco M, Bisley J, Grundfest W, Dutson E. Pneumatic balloon actuators for tactile feedback in robotic surgery. Ind Robot An Int J 2008;35:449-55.

[10] Tactus Technology I. Tactus Technology 2012.

[11] Nara T, Takasaki M, Maeda T, Higuchi T, Ando S, Tachi S. Surface acoustic wave tactile display. IEEE Comput Graph Appl 2001;21:56-63.

[12] Gavrilov LR, Tsirulnikov EM, Davies IA. Application of focused ultrasound for the stimulation of neural structures. Ultrasound Med Biol 1996;22:179-92.

[13] Khaled W, Reichling S, Bruhns OT, Boese H, Baumann M, Monkman G, et al. Palpation imaging using a haptic system for virtual reality applications in medicine. Stud Health Technol Inform 2004;98:147-53.

[14] Gregory J, Shen Y, Xi N. Stimulation Current Control for Load-Aware Electrotactile Haptic Rendering: Modeling and Simulation. Int Conf Robot Autom 2011.

[15] Kim Y, Oakley I, Ryu J. Design and psychophysical evaluation of pneumatic tactile display. SICE-ICASE, 2006. Int. Jt. Conf., 2006, p. 1933-8.

[16] Liu Y, Davidson R, Taylor P, Ngu J, Zarraga J. Single cell magnetorheological fluid based tactile display. Displays 2005;26:29-35. 
Min Li, Tommaso Ranzani, Sina Sareh, Lakmal D. Seneviratne, Prokar Dasgupta, Helge A Wurdemann and Kaspar Althoefer 2014 Multi-Fingered Haptic Palpation utilizing Granular Jamming Stiffness Feedback Actuators Smart Mater. Struct. 23 095007 doi:10.1088/0964-1726/23/9/095007

[17] Langrana NA, Burdea G, Lange K, Gomez D, Deshpande S. Dynamic force feedback in a virtual knee palpation. Artif Intell Med 1994;6:321-33.

[18] Dinsmore M, Langrana N, Burdea G, Ladeji J. Virtual reality training simulation for palpation of subsurface tumors. IEEE Virtual Real. Annu. Int. Symp., IEEE Computer Society; 1997, p. $54-60$.

[19] Daniulaitis V, Alhalabi MO. Medical palpation of deformable tissue using physics-based model for gaptic interface robot ( HIRO ). Inf. Syst., 2004, p. 3907-11.

[20] Endo T, Kawasaki H, Mouri T, Doi Y, Yoshida T, Ishigure Y, et al. Five-fingered haptic interface robot: HIRO III. World Haptics 2009 Third Jt EuroHaptics Conf Symp Haptic Interfaces Virtual Environ Teleoperator Syst 2009;4:458-63.

[21] Yalcintas M, Dai H. Magnetorheological and electrorheological materials in adaptive structures and their performance comparison. Smart Mater Struct 1999;8:560-73.

[22] Liu AJ, Nagel SR. Jamming is not just cool any more. Nature 1998;396:21-2.

[23] Mitsuda T, Kuge S, Wakabayashi M, Kawamura S. Wearable haptic display by the use of a Particle Mechanical Constraint. IEEE Comput. Soc; 2002.

[24] Brown E, Rodenberg N, Amend J, Mozeika A, Steltz E, Zakin MR, et al. Universal robotic gripper based on the jamming of granular material. Proc Natl Acad Sci U S A 2010;107:18809-14.

[25] Cheng NG, Lobovsky MB, Keating SJ, Setapen AM, Gero KI, Hosoi AE, et al. Design and analysis of a robust, low-cost, highly articulated manipulator enabled by jamming of granular media. Proc. - IEEE Int. Conf. Robot. Autom., 2012, p. 4328-33.

[26] Steltz E, Mozeika A, Rembisz J, Corson N, Jaeger HM. Jamming as an Enabling Technology for Soft Robotics. SPIE 2010;7642:764225.

[27] Jiang A, Xynogalas G, Dasgupta P, Althoefer K, Nanayakkara T. Design of a variable stiffness flexible manipulator with composite granular jamming and membrane coupling. 2012 IEEE/RSJ Int. Conf. Intell. Robot. Syst., IEEE; 2012, p. 2922-7.

[28] Cianchetti M, Ranzani T, Gerboni G, De Falco I, Laschi C, Menciassi A. STIFF-FLOP Surgical Manipulator: mechanical design and experimental characterization of the single module. IEEE/RSJ Int. Conf. Intell. Robot. Syst., 2013.

[29] Jiang A, Ranzani T, Gerboni G, Lekstutyte L, Althoefer K, Dasgupta P, et al. Robotic granular jamming: Does the membrane matter? Soft Robot 2014:Accepted.

[30] Li M, Luo S, Seneviratne LD, Nanayakkara T, Dasgupta P, Althoefer KA. Haptics for multifingered palpation. IEEE Int. Conf. Syst. Man, Cybern., 2013.

[31] Wu JZ, Welcome DE, Dong RG. Three-dimensional finite element simulations of the mechanical response of the fingertip to static and dynamic compressions. Comput Methods Biomech Biomed Engin 2006;9:55-63.

[32] Klemm D. Fingertip anatomy n.d.

[33] Yamada H. Strength of Biological Materials. Baltimore, MD: Willians and Wilkins Co.; 1970.

[34] Wayne RO. Plant cell biology. Academic Press; 2009.

[35] Smooth-on Inc. Technical overview of Ecoflex Series 2014:1.

[36] Wu JZ, Krajnak K, Welcome DE, Dong RG. Analysis of the dynamic strains in a fingertip exposed to vibrations: Correlation to the mechanical stimuli on mechanoreceptors. J Biomech 2006;39:2445-56.

[37] Altman D, Bland J. Diagnostic test 1: Sensitivity and specificity. BMJ 1994;308:1552.

[38] Fawcett T. An introduction to ROC analysis. Pattern Recognit Lett 2006;27:861-74.

[39] Wilson EB. Probable inference, the law of succession, and statistical inference. J Am Stat Assoc 1927;22:209-12.

[40] Wallis S. Binomial confidence intervals and contingency tests: mathematical fundamentals and the evaluation of alternative methods. J Quant Linguist 2013;20:178-208. 